\title{
Successful ICU Care in a MERRF Patient with Severe Covid-19
}

\section{Scott Clanton $I^{1 *}$, Rajani Adiga, MS ${ }^{2}$, Amy Christie, MD, FACS ${ }^{3,4 t}$ and Dennis Ashley, MD, FACS, FCCM ${ }^{5,6 \#}$}

${ }^{1}$ Medical Student, Mercer University School of Medicine, USA

${ }^{2}$ Research Coordinator, Trauma Services at Atrium Health Navicent, USA

${ }^{3}$ Director of Adult Critical Care, Department of Critical Care, Atrium Health Navicent, USA

${ }^{4}$ Assistant Professor Surgery, Department of Surgery, Mercer University School of Medicine, USA

${ }^{5}$ Director of Trauma and Adult Critical Care, Atrium Health Navicent, USA

${ }^{6}$ Professor of Surgery, Department of Surgery, Mercer University School of Medicine, USA

${ }^{\#}$ Equal Contribution

*Corresponding author: Scott Clanton II, Medical Student, Mercer University School of Medicine, 3300 N Ingle PI \#9D, Macon, GA 31210, USA

\begin{abstract}
Mitochondrial myopathies are a spectrum of rare diseases caused by a genetic defect in ATP production at the level of the electron transport chain. The degree of disability is variable, but often patients are severely ill and highly susceptible to increased morbidity and mortality with infection. Covid-19 is known to be especially virulent in patients with significant comorbidities. In this case, a 27-year-old man with Myoclonus Epilepsy with Ragged Red Fibers (MERRF) presented in severe shock due to Covid-19 infection with bacterial pneumonia co-infection. The patient was successfully treated and discharged following prolonged intubation and deconditioning. This case illustrates the severity of presentation in MERRF patients with Covid-19 as well as the effectiveness of early intervention.
\end{abstract}

\section{Introduction}

Mitochondrial Myopathies are a spectrum of maternally inherited genetic syndromes typically caused by point mutations in mitochondrial DNA that cause disruptions in mitochondrial membranes [1]. The structure and function of mitochondrial membranes are intrinsically linked, and therefore the primary etiology of these conditions is typically a disruption in oxidative phosphorylation, an essential source of ATP [2]. A commonality of these syndromes includes disruption of skeletal muscle fibers into "ragged" fibers, due to the high ATP utilization in these tissues as well as their high density of mitochondria [1,2]. Beyond ATP generation, mitochondria play an essential role in calcium regulation, apoptosis, heme synthesis, inflammation, and nuclear gene regulation [2]. These aberrations in mitochondrial function cause a range of syndromes.

Myoclonic Epilepsy with Ragged Red Fibers (MERRF) is one such syndrome caused by the m.8344A > $G$ substitution in the MT-TK gene, responsible for producing tRNA $A^{\text {lys }}$, essential in the transfer of lysine residues during translation [3]. This mutation is present in $~ 80 \%$ of clinically diagnosed MERRF [4]. Clinically, the syndrome is commonly characterized by raggedred fiber myopathy, cardiac arrythmia due to structural abnormalities, myoclonic epilepsy, cerebellar ataxia, dementia, sensorineural hearing loss, stroke-like events, and optic atrophy $[3,4]$. Like most mitochondrial myopathies, MERRF syndromes are phenotypically heterogeneous, and can include a wide spectrum of symptomology involving the CNS, PNS, cardiovascular system, Gl system, or endocrine system [1,5]. Lifethreatening complications include status epilepticus, respiratory weakness, pancreatitis, lactic acidosis, and cardiac arrythmia or cardiomyopathy $[1,6]$. Management of this condition is complex, but primarily focuses on seizure prevention and control while avoiding antiepileptics that are toxic to mitochondria [6]. Overall prognosis is poor, but variable based on phenotype [3].

Covid-19 is an emerging viral illness first identified in 
2019 in Wuhan, China that can cause severe illness in the presence of significant comorbidities $[7,8]$. Cough, fever, dyspnea, are the prevailing symptoms, and this respiratory distress can lead to Acute Respiratory Distress Syndrome (ARDS), with the presence and severity of dyspnea being the most accurate predictor of outcomes discovered so far [8]. Common comorbidities such as diabetes, obesity, COPD, hypertension, cardiovascular disease, Liver disease, and renal failure have been shown to cause significantly increased morbidity in Covid-19 patients $[7,8]$.

Recovery is often slow and incomplete following severe disease, leading to prolonged hospital and ICU stays [9]. There have been select case reports on myositis, possibly dermatomyositis, present following Covid-19 infection, suggesting the possibility of muscular involvement caused by this infection [10].

The possibility of severe phenotypes of MERRF syndrome coupled with the high possibility of severe Covid-19 infection in those with significant comorbidities warrants special consideration in the management of patients with mitochondrial myopathies such as MERRF syndrome. Of particular interest are the overlapping respiratory insufficiency present in both, leading to compounding muscular dysfunction of muscles of respiration as well as the intrinsic lung disruption [1,9]. The following case illustrates such a complication.

\section{Case Report}

$\mathrm{CH}$ is a 27-year-old male with a history of MERRF syndrome with epilepsy, pigmentary retinal dystrophy, and multiple myocardial infarctions with an implanted cardio defibrillator, and dysphagia with G-tube dependence who presented to the emergency department in May of 2020 as a transfer from a skilled nursing facility with respiratory distress, severe hypotension, and tachycardia in the range of 150160 bpm with unknown time of onset. The patient required immediate intubation on $100 \% \mathrm{O}_{2}$ in the ED. He also received a $2.5 \mathrm{~L}$ fluid bolus and was started on broad-spectrum antibiotics. Initial laboratory workup revealed anion gap metabolic acidosis with elevated lactate of 10.9, hypernatremia, leukocytosis with a total WBC count of 25.71, elevated BNP to 4417, and elevated troponin above baseline at 1.335. Imaging showed diffuse ground-glass opacities in both lungs on CTA (Figure 1) and advanced cerebral atrophy with ventriculomegaly on a contrast head CT consistent with the patients known intellectual disability. Microbiology showed a positive rapid Covid-19 test and a bronchi alveolar lavage positive for Haemophilus influenzae. ECG showed diffuse ST depression without clear localization. Initial management included immediate transfer to the cardiovascular ICU, continuous fluid resuscitation, vasopressor support, continuation of broad-spectrum antibiotics (aztreonam, vancomycin), continuous EEG monitoring for seizure activity, and propofol for pain control.

The patient responded well to ventilation and was weaned to $40 \% \mathrm{FiO}_{2}$ with PEEP of $8 \mathrm{cmH}_{2} \mathrm{O}$ over 24 hours. Troponins trended down as well over the same time frame. Seizure activity was regularly observed over this time and the patient received unfractionated heparin for DVT prophylaxis. Upon receiving cultures and sensitivities, the antibiotic regimen was changed to IV ceftriaxone over 7 days. Appropriate vitamin replacement was restarted for continuous management of MERRF lamotrigine $2000 \mathrm{mg}$ BID, valproic acid 500 mg QID, and clonazepam $1 \mathrm{mg}$ BID were restarted for chronic management of epilepsy.

The patient remained stable and began to respond to stimuli on ICU day 5. Spontaneous breathing trials were initiated on day 6 , however the patient remained ventilated on $30 \% \mathrm{O}_{2}$. Seizure activity was not observed again until day 13 . The patient was extubated on day 16 to HFNC on $50 \% \mathrm{FiO}_{2}$ with a flow rate of $6 \mathrm{~L}$. Repeat BAL grew Staphylococcus aureus and the patient was started on vancomycin, however the patient remained extubated. On hospital day 22, the patient was transferred to the hospital floor from the ICU due to improved tachycardia and decreased $\mathrm{O}_{2}$ demand. On day 23, the patient had a seizure episode with tachypnea and tachycardia and was re-admitted to the ICU and was re-intubated with a PEEP of $8 \mathrm{~cm} \mathrm{Hg}$ and $40 \% \mathrm{O}_{2}$. The following day the patient was weaned back to CPAP. On day 30, the patient was transferred back to the hospital floor. A PEG tube was inserted on day 37 due to dysphagia. The remainder of the stay was supportive with no significant events. The patient's mental status slowly returned to his baseline, opening his eyes and responding to his name. The patient was discharged to a skilled nursing facility on hospital day 77 following return to baseline mental status. The patient persistently tested positive for Covid-19 over the entire stay. Of note, the patient did not receive remdesivir or dexamethasone, as this was not yet the standard of care at the time of his stay.

\section{Discussion}

MERRF syndrome patients often have profound dysfunction and are especially susceptible to infectious insult. With the emergence of Covid-19, known for its high morbidity and mortality rates in those with significant comorbidities, careful attention must be paid to patients with rare and severe genetic diseases such as mitochondrial myopathies $[8,9]$. This case demonstrates the severe sequalae that can occur in a MERRF syndrome patient with Covid-19 infection.

This patient was difficult to treat due to a low seizure threshold, fragile cardiac myocytes leading to diffuse cardiac ischemia, and coinfection with Haemophilus influenzae. Management of seizure 
activity was critical in this case, as a single seizure episode led to re-intubation and transport back to the ICU. The patient was successfully treated, with the most notable interventions in this case being early intubation with careful weaning and early antibiotics, as well as presser and fluid support for shock. With the persistent prevalence of Covid-19 infection becoming more and more likely, management of patients such as this one will continue to be a challenge for the future. Due to their low prevalence, patients with mitochondrial myopathies are poorly studied, and therefore developing standardized treatment for these cases is difficult.

\section{Conclusions}

Covid-19 infection in the context of mitochondrial myopathies can have a severe presentation, and early intervention that includes management of chronic symptoms is likely to be effective in treatment.

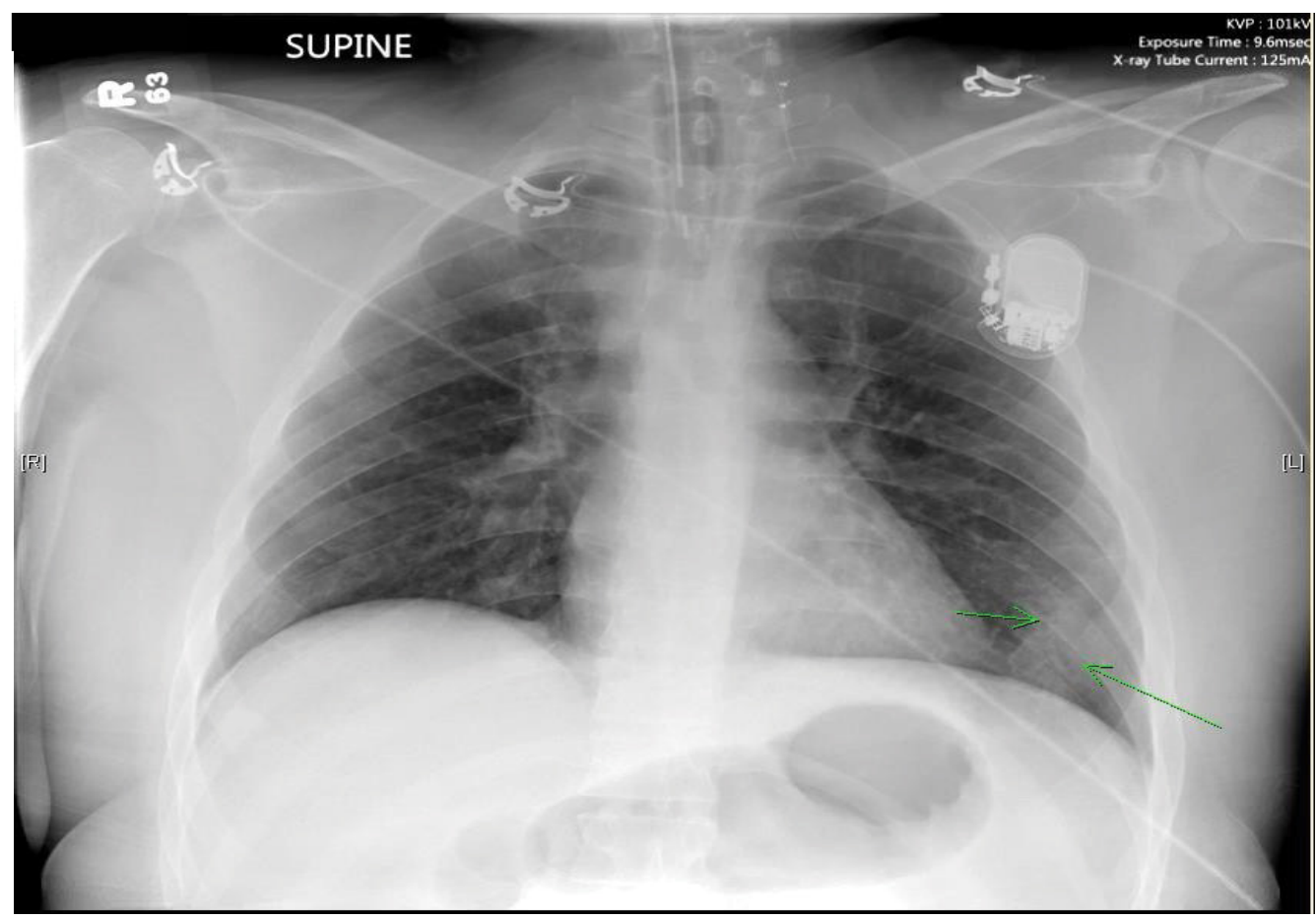

Day 0, portable chest X-ray. Impression: Endotracheal tube projects in expected location. Battery pack is noted over the left chest with leads extending into the left neck. The cardiomediastinal silhouette is normal. The lungs are clear other than a small focus of infiltrate in the left lower lung. There is no appreciable pleural effusion or pneumothorax.

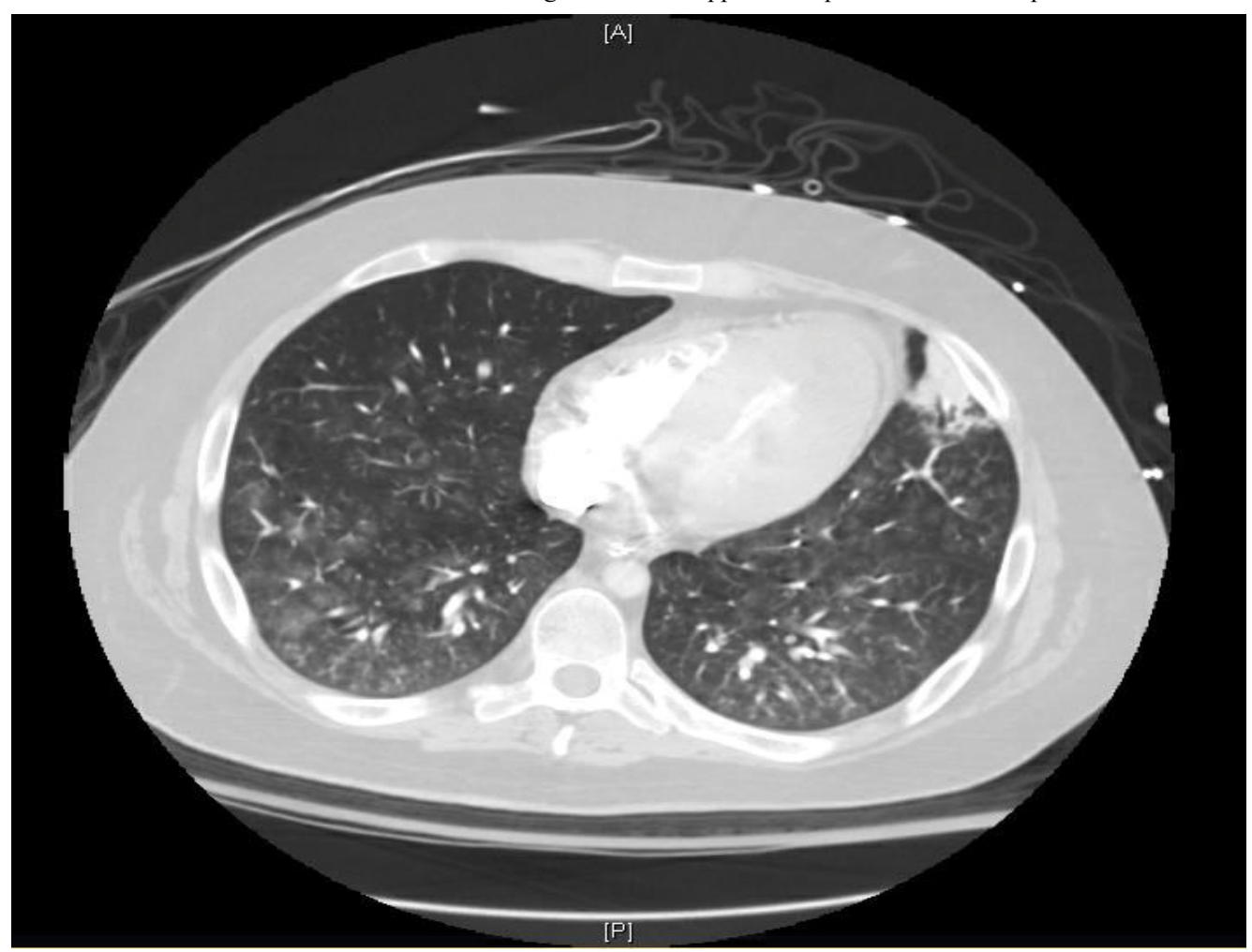




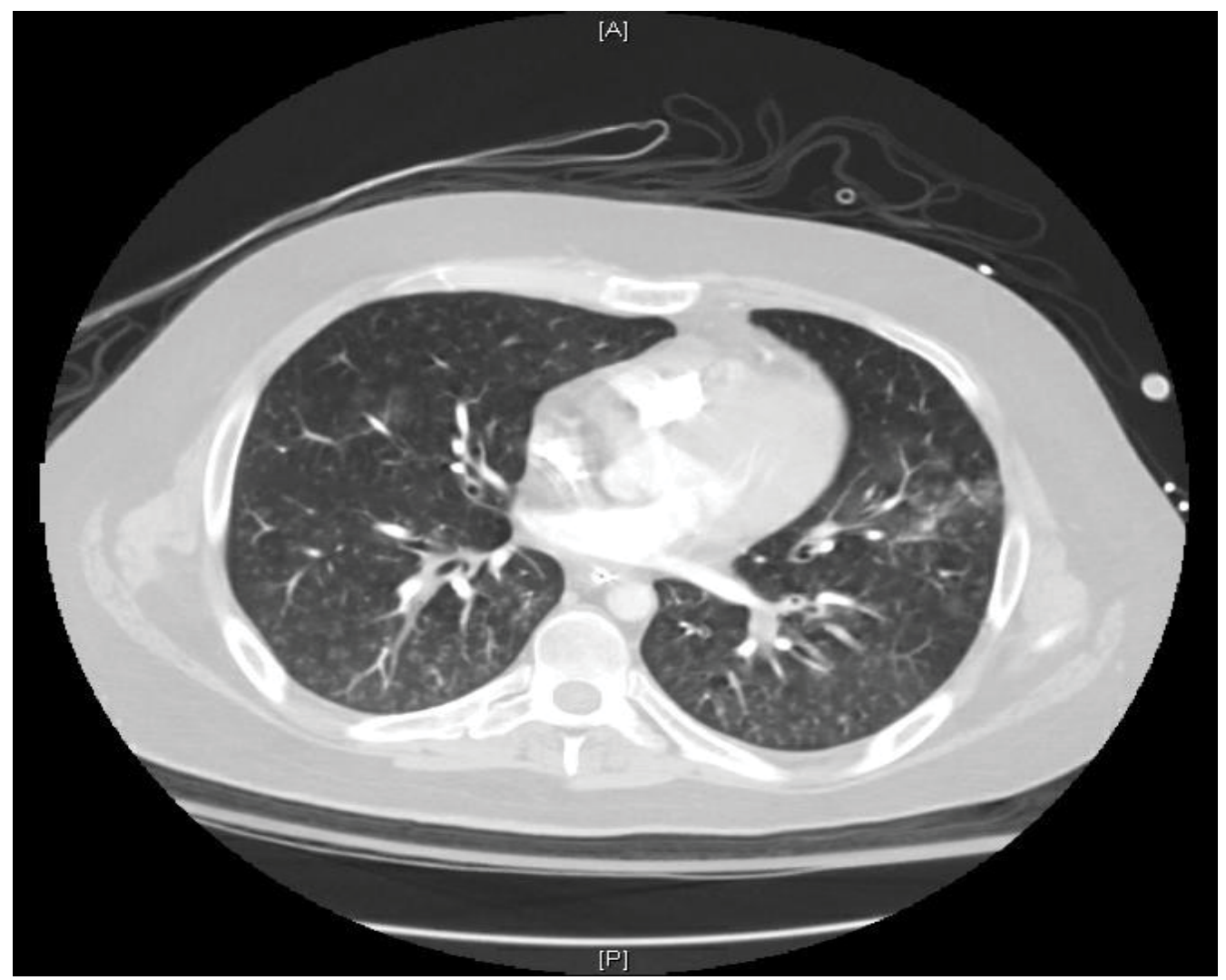

Day 0, CTA, There are diffuse groundglass and tree-in-bud opacities throughout the lungs, greatest in the lung bases. There is a wedge-shaped area of consolidation in the lingula and in the right lower lobe. There is no pleural effusion.

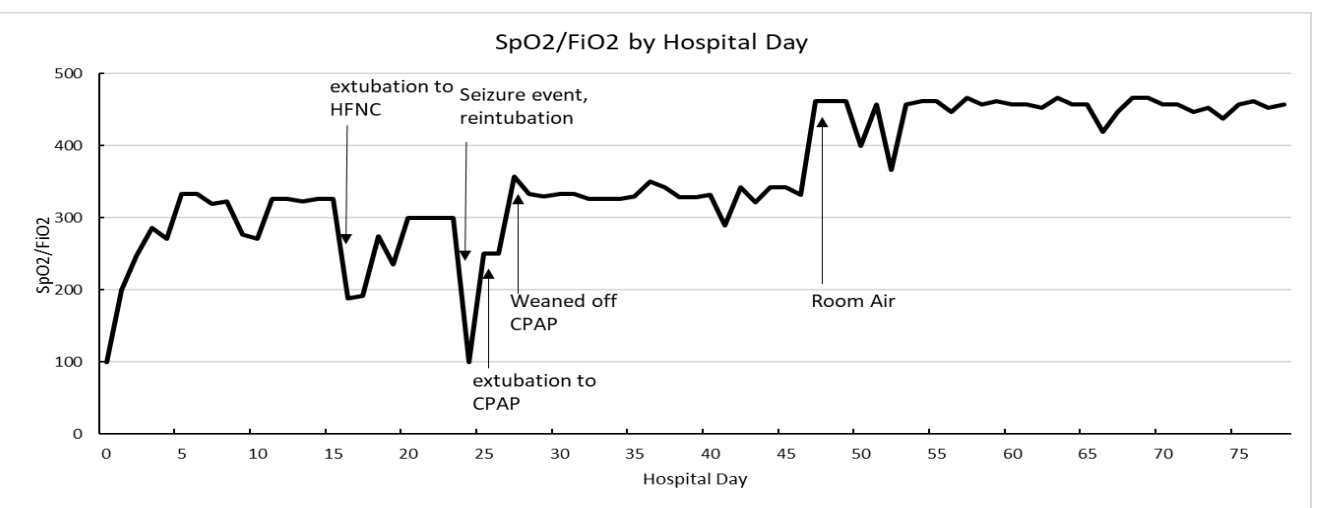

Figure 1: The patient's respiratory status is measured above as a ratio of $\mathrm{SpO}_{2} / \mathrm{FiO}_{2}$, an approximation of $\mathrm{PaO}_{2} / \mathrm{FiO}_{2}$ or the P:F ratio. The patient was intubated on day 0 , extubated to HFNC on day 15 , reduced to NC on day 18 , reintubated on day 24, weaned to CPAP on day 25, reduced to NC on day 27, and was off $\mathrm{O}_{2}$ support entirely on day 47. Mild Acute Respiratory Distress Syndrome (ARDS) is defined as P:F ratio < 300, moderate as $<200$, and severe as $<100$. As seen above, the patient presented in severe ARDS which quickly improved to mild with treatment. Seizure activity caused a severe syndrome as well, which also quickly responded to intervention. The patient ultimately made a full recovery from ARDS.

\section{Funding}

This work was supported by an unrestricted research grant from the Navicent Health Foundation. The CRF was part of The Viral Infection and Respiratory Illness Universal Study (VIRUS), a prospective cohort study and registry of all eligible adult and pediatric patients who were admitted to a hospital due to COVID-19 [11,12].

\section{References}

1. Finsterer J, Zarrouk-Mahjoub S, SHoffner JM (2021) MERRF Classification: Implications for diagnosis and clinical trials. Pediatric Neurol 80: 8-23.

2. Vincent $A E, N g$ YS, White K, Davey T, Mannella C, et al. (2016) The spectrum of mitochondrial ultrastructural defects in mitochondrial myopathy. Scientific Reports 6 .

3. Hameed S, Tadi P (2021) Myoclonic Epilepsy and Ragged Red Fibers (MERF). StatPearls.

4. Quadir A, Pontifex CS, Robertson HL, Labos C, Pfeffer G (2019) Systematic review and meta-analysis of cardiac involvement in mitochondrial myopathy. Neurology Genetics 5.

5. Mancuso M, Orsucci D, Angelini C, Bertini E, Carelli V, et al. 
(2013) Phenotypic heterogeneity of the 8344A>G mtDNA "MERRF" mutation. Neurology 80: 2049-2054.

6. Finsterer J, Zarrouk-Mahjoub S (2017) Management of epilepsy in MERRF syndrome. Seizure 50: 166-170.

7. Jain V, Yuan JM (2020) Predictive symptoms and comorbidities for severe COVID-19 and intensive care unit admission: a systematic review and meta-analysis. Int $\mathrm{J}$ Public Health 65: 533-546.

8. Ejaz $\mathrm{H}$, Alsrhani $\mathrm{A}$, Zafar $\mathrm{A}$, Javed $\mathrm{H}$, Junaid $\mathrm{K}$, et al (2020) COVID-19 and comorbidities: Deleterious impact on infected patients. J Infect Public Health 13: 1833-1839.

9. Rooney S, Webster A, Paul L (2020) Systematic review of changes and recovery in physical function and fitness after severe acute respiratory syndrome-related coronavirus infection: Implications for COVID-19 rehabilitation. Phys Ther 100: 1717-1729.
10. Zhang $\mathrm{H}$, Charmchi Z, Seidman RJ, Anziska $\mathrm{Y}$, Velayudhan V, et al. (2020) COVID-19-associated myositis with severe proximal and bulbar weakness. Muscle Nerve 62: E57-E60.

11. Walkey AJ, Kumar VK, Harhay MO, Bolesta S, Bansal V, et al. (2020) The viral infection and respiratory illness universal study (virus): an international registry of coronavirus 2019-related critical illness. Crit Care Explor 2: p e0113.

12. Walkey AJ, Sheldrick RC, Kashyap R, Kumar VK, Boman $\mathrm{K}$, et al.(2020) Guiding principles for the conduct of observational critical care research for coronavirus disease 2019 pandemics and beyond: the society of critical care medicine discovery viral infection and respiratory illness universal study registry. Crit Care Med 48: e1038-e1044 